\title{
Brane cosmology with a bulk scalar field
}

\author{
David Langlois, María Rodríguez-Martínez \\ Institut d'Astrophysique de Paris, \\ (Centre National de la Recherche Scientifique) \\ 98bis Boulevard Arago, 75014 Paris, France
}

(October 20, 2018)

\begin{abstract}
We consider "cosmologically symmetric" (i.e. solutions with homogeneity and isotropy along three spatial dimensions) five-dimensional spacetimes with a scalar field and a three-brane representing our universe. We write Einstein's equations in a conformal gauge, using light-cone coordinates. We obtain explicit solutions: a. assuming proportionality between the scalar field and the logarithm of the (bulk) scale factor; b. assuming separable solutions. We then discuss the cosmology in the brane induced by these solutions.
\end{abstract}

\section{INTRODUCTION}

The idea that our world might be a brane embedded in a higher dimensional spacetime has generated lately an intensive research, notably in cosmology. In general, the confinement of matter on the brane, leads to a cosmological evolution in the brane which is different from the usual evolution governed by Friedmann's law [1].

Such a deviation is problematic for the "recent" history of the universe, since the usual nucleosynthesis scenario would not work anymore. The simplest way to cure this problem [2] is to introduce a negative cosmological constant in the bulk, à la Randall-Sundrum [3], and usual cosmological evolution is recovered at late times when the energy density 
of cosmological matter $\rho$ is much smaller than the brane tension $\sigma$ (fine-tuned so as to compensate the bulk cosmological constant) [4.5].

This solution, however, requires a fine-tuning between the brane tension and the bulk cosmological constant and it would be desirable to obtain models where this requirement can be evaded. This suggests the study of more complicated models containing dynamical fields in the bulk.

As a first step, it is natural to consider the presence of a scalar field in the bulk. This possibility has already been investigated in several works, for various motivations. One of the first motivations to introduce a bulk scalar field was to stabilize [6] the distance between the two branes in the context of the first model introduced by Randall and Sundrum [7]. A second, more recent, motivation for studying scalar fields in the bulk was the possibility that such a set-up could provide some clue to solve the famous cosmological constant problem 8.

In this perspective, some effort has been devoted to the construction of cosmological solutions, i.e. with time evolution in the brane, with a scalar field in the bulk [9 12] (see also the earlier solutions of [13]). Several works have studied in particular the impact of the presence of a scalar field in the bulk on the cosmological evolution in the brane, without trying to solve the full system of equations in the bulk [14 17]. However, if one wishes to evaluate quantitatively, and not only qualitatively, the impact of the bulk scalar field on the brane evolution, one needs in general a solution of the bulk equations. It is the purpose of the present work to provide some solutions of the full system of equations and to study the corresponding brane evolution. The general problem being rather difficult, it will be seen that the requirement of obtaining exact analytical solutions is not easy to reconcile with the wish to obtain realistic cosmological solutions. But we hope our solutions might be helpful to give some insights for the more general situation.

In the present work, we have tried to obtain explicit solutions, by using the conformal gauge and light-like coordinates, a technique which has been employed for instance to the case of an empty bulk (but with a cosmological constant) [18 or in the case of a bulk with a 
scalar field, like here, but with a vanishing potential [9]. We have managed to obtain explicit solutions by imposing two types of ansätze. A first ansatz, very powerful to integrate the full system of Einstein's equations, will be to assume a special relation between the scalar field and the bulk scale factor. As we will show, it turns out that the bulk solution is then necessarily static and one recovers some of the solutions obtained by [13]. A second ansatz will be to assume that the solutions are separable. In both cases, one must consider an exponential potential for the scalar field.

The plan of the paper is the following. In the second section, we introduce the general set-up and write down the full system of equations. In the third section, we study the solutions obtained with the additional constraint between the scalar field and the metric mentioned above. We then consider, in the fourth section, the case of separable solutions. The fifth section is devoted to the cosmological evolution in the brane induced by the various bulk solutions we have found. We finally conclude in the last section.

\section{THE MODEL}

Let us first define our set-up. We consider a scalar field $\phi$ living in a five-dimensional spacetime endowed with the metric $g_{A B}$. We also assume the existence of three-brane in this spacetime in which unspecified matter is confined. The action will be taken to be of the form

$$
\mathcal{S}=\int d^{5} x \sqrt{-g}\left[\frac{1}{2 \kappa^{2}}{ }^{(5)} R-\frac{1}{2}\left(\nabla_{A} \phi\right) \nabla^{A} \phi-V(\phi)\right]+\int d^{4} x \mathcal{L}_{4}
$$

where ${ }^{(5)} R$ is the scalar curvature of the five-dimensional metric $g_{A B}$ and where the second term is the action for the brane, which we leave unspecified at this stage, but which is assumed to depend on the bulk scalar field in general.

Since we consider only cosmological solutions, we will assume that the metric is isotropic and homogeneous along the three ordinary spatial dimensions. Choosing the so-called conformal gauge, the metric then reads 


$$
d s^{2}=g_{A B} d x^{A} d x^{B}=e^{2 B(t, y)}\left(-d t^{2}+d y^{2}\right)+e^{2 A(t, y)} \delta_{i j} d x^{i} d x^{j},
$$

where we have supposed flat three-dimensional subspaces for simplicity. Introducing the light-cone coordinates, $u \equiv t-y$ and $v \equiv t+y$, the metric can be rewritten in the form

$$
d s^{2}=-e^{2 B(u, v)} d u d v+e^{2 A(u, v)} \delta_{i j} d x^{i} d x^{j} .
$$

The matter content of the five-dimensional spacetime is described by the energymomentum tensor, which can be derived from the action (1). The total energy-momentum tensor is then found to be of the form,

$$
T_{A B}=\partial_{A} \phi \partial_{B} \phi-g_{A B}\left[\frac{1}{2}\left(\nabla_{C} \phi\right)\left(\nabla^{C} \phi\right)+V(\phi)\right]+T_{A B}^{\text {brane }},
$$

the first part coming from bulk scalar field, the second part being confined in the brane, which will be assumed to lie at $y=0$.

In the light-cone coordinates, the five-dimensional Einstein's equations, which follow from the variation of the above action (1) with respect to the five-dimensional metric, reduce, in the bulk to the following set of equations:

$$
\begin{aligned}
A_{, u v}+3 A_{, v} A_{, u} & =\frac{\kappa^{2}}{6} e^{2 B} V(\phi) \\
-3 A_{, u u}+6 A_{, u} B_{, u}-3 A_{, u}{ }^{2} & =\kappa^{2} \phi_{, u}{ }^{2} \\
-3 A_{, v v}+6 A_{, v} B_{, v}-3 A_{, v}{ }^{2} & =\kappa^{2} \phi_{, v}{ }^{2} \\
B_{, u v}+2 A_{, u v}+3 A_{, v} A_{, u} & =-\frac{1}{2} \kappa^{2} \phi_{, v} \phi_{, u}+\frac{\kappa^{2}}{4} e^{2 B} V(\phi) .
\end{aligned}
$$

Here, (5) is the $u v$ component of Eintein's equations, (6) and (7) are the $u u$ and $v v$ components respectively; (8) comes from the ordinary spatial components. Finally, the KleinGordon equation in the bulk reads

$$
\phi_{, v} A_{, u}+A_{, v} \phi_{, u}+\frac{2}{3} \phi_{, u v}=-\frac{1}{6} e^{2 B} V^{\prime}(\phi) .
$$

Of course, there is some redundancy in the system of equations (5-9) because of Bianchi's identities. If one considers a free scalar field in the bulk, i.e. without potential, then 
our system of equations reduces exactly to that written by Horowitz, Low and Zee [9]. When the potential is non vanishing, by contrast, solutions such that $A=A(u)$ or $A=$ $A(v)$, repectively purely outgoing and ingoing waves, are no longer possible, simply because ingoing/outgoing waves will be scattered by the potential.

The above system of equations applies only for the bulk and must now be completed by boundary conditions at the location of the brane. An alternative, equivalent, procedure would be to write down the complete Einstein and Klein-Gordon equations, with distributional source terms representing the brane. Let us start with the boundary conditions for the metric components, $A$ and $B$. They follow from the Darmois-Israel junction conditions, which, for brane-universes, take the form [1]:

$$
\left[K_{\mu \nu}\right]=-\kappa^{2}\left(S_{\mu \nu}-\frac{1}{3} S g_{\mu \nu}\right)
$$

where $K_{\mu \nu}$ is the extrinsic curvature tensor associated to the brane, and where $S_{\mu \nu}$ is the four-dimensional energy-momentum tensor of the matter confined in the brane, which is obtained from the variation of the brane action with respect to the induced metric $g_{\mu \nu}$. Since we investigate only cosmological solutions, i.e. homogeneous and isotropic with respect to the three ordinary spatial dimensions, $S_{\mu \nu}$ is necessarily of the perfect fluid form:

$$
S_{\mu \nu}=(\rho+P) u_{\mu} u_{\nu}+P g_{\mu \nu}
$$

where $\rho$ is the energy density, $P$ the pressure, and $u^{\mu}$ the unit time-like vector normal to the homogeneous and isotropic 3-surfaces.

Expressing the extrinsic curvature tensor components in terms of the metric components, and assuming the usual mirror symmetry with respect to the brane, the junction conditions (10) yield the two following equations,

$$
\begin{aligned}
& \left.\frac{\partial A(y, t)}{\partial y}\right|_{y=0}=-\frac{\kappa^{2}}{6} e^{B} \rho(t) \\
& \left.\frac{\partial B(y, t)}{\partial y}\right|_{y=0}=\frac{\kappa^{2}}{6} e^{B}(3 P(t)+2 \rho(t)) .
\end{aligned}
$$


Let us now turn to the boundary condition for the scalar field. It is related to the variation of the brane Lagrangian with respect to the scalar field. An explicit boundary condition for $\phi$ thus requires the specification of the dependence of $\mathcal{L}_{4}$ with respect to $\phi$. Several possibilities have been considered in the literature (see [17 for a detailed discussion). A first possibility (see e.g. [14) is to assume that the brane matter is minimally coupled to a metric $\tilde{g}_{\mu \nu}$, which is conformally related to the metric defined in the bulk action, i.e.

$$
\tilde{g}_{\mu \nu}=e^{2 k(\phi)} g_{\mu \nu}
$$

In this case, one can define an energy-momentum tensor different from $S_{\mu \nu}$ by taking the variation of the brane action with respect to $\tilde{g}_{\mu \nu}$ instead of $g_{\mu \nu}$, namely

$$
\tilde{S}^{\mu \nu}=\frac{2}{\sqrt{-\tilde{g}}} \frac{\delta S_{m}}{\delta \tilde{g}_{\mu \nu}} .
$$

The corresponding energy density $\tilde{\rho}$ and $\tilde{P}$ are related to the former ones by the relations

$$
\rho=e^{4 k(\phi)} \tilde{\rho}, \quad P=e^{4 k(\phi)} \tilde{P} .
$$

Variation of the global action with respect to $\phi$ leads to a Klein-Gordon equation of the form

$$
\nabla_{A} \nabla^{A} \phi=\frac{d V}{d \phi}-e^{-B} k^{\prime}(\phi) S \delta(y)
$$

where $S \equiv-\rho+3 P$ is the trace of the energy-momentum tensor. This means that the scalar field must satisfy the equation (9) given above in the bulk as well as the boundary condition at the brane location $y=0$ :

$$
\left.\frac{\partial \phi(y, t)}{\partial y}\right|_{y=0}=-\frac{1}{2} e^{B} k^{\prime}(\phi) S .
$$

For simplicity, we will assume in the following that $k(\phi)=\chi \tilde{\phi}$, where we have introduced the dimensionless rescaled scalar field,

$$
\tilde{\phi}=\frac{\kappa}{\sqrt{3}} \phi
$$


which it will often be convenient to use.

Another possibility (see e.g. [15]), named volume-element coupling in [17], is to consider an overall function of $\phi$ that multiplies the Lagrangian density so that

$$
\mathcal{L}_{4}=e^{4 \chi \tilde{\phi}} \mathcal{L}\left(g_{\mu \nu}, \psi_{i}\right)
$$

where the $\psi_{i}$ are all matter fields that are confined in the brane. In practice, in order to study the cosmological evolution in the brane, one wishes an effective description of the matter in the brane and a perfect fluid is quite appropriate for this purpose. However, a perfect fluid is an effective matter for which there is no natural fundamental description in terms of a Lagrangian density. There is nevertheless a substantial literature on a Lagrangian description of relativistic perfect fluids and various formulations exist. If all these formulations yield the same equations of motion, they are not equivalent at the Lagrangian level. This means, starting from (20), different formulations will yield different couplings between the perfect fluid matter and the bulk scalar field. For instance, 15 have used a Clebsh-Jordan formulation where the Lagrangian density is given by the pressure, whereas [9] have used a Taub type approach where the Lagrangian density is proportional to the energy density. These formulations are equivalent only when the equation of state is $P=-\rho$, i.e. if the matter behaves like a cosmological constant, but they give different couplings in all other cases.

In practice, we will use a boundary condition of the form

$$
\left.e^{-B} \frac{\partial \tilde{\phi}(y, t)}{\partial y}\right|_{y=0}=\frac{\kappa^{2}}{6} \gamma \rho(t)
$$

which involves all cases where the equation of state for matter is of the form $P=w \rho$, with $w$ constant. In the case of conformal coupling, the expression for $\gamma$ is given by

$$
\gamma=-(3 w-1) \chi
$$

In the case of volume-element coupling, one would get

$$
\gamma=4 \chi
$$


if the Lagrangian density for the perfect fluid is proportional to the energy density as in [9], or

$$
\gamma=-4 w \chi
$$

if the Lagrangian density is proportional to the pressure as was chosen in [15] or [17]. If the matter content of the brane behaves like a cosmological constant, i.e. $w=-1$, the three above expressions yield, as expected, the same result, and there is no ambiguity.

\section{III. "PROPORTIONAL" SOLUTIONS}

In this section, we will solve the Einstein and Klein-Gordon equations with the assumption that

$$
\tilde{\phi}(u, v)=\lambda A(u, v) .
$$

Substituting this ansatz in the $u v$ component of Einstein's equations (5) as well as in the Klein-Gordon equation (9), one finds that the bulk potential for the scalar field is necessarily of the exponential form with

$$
V(\phi)=V_{0} e^{-2 \lambda \tilde{\phi}}
$$

\section{A. Bulk equations}

The $(u u)$ and $(v v)$ components of Einstein's equations, i.e. (6) and (7), after substitution of (25), yield the following relations:

$$
\begin{aligned}
2 B_{, u} & =\left(1+\lambda^{2}\right) A_{, u}+\frac{A_{, u u}}{A_{, u}} \\
2 B_{, v} & =\left(1+\lambda^{2}\right) A_{, v}+\frac{A_{, v v}}{A_{, v}} .
\end{aligned}
$$

These equations imply that $A(u, v)$ is necessarily of the form 


$$
A(u, v)=f(\tilde{U}(u)+\tilde{V}(v))
$$

where $f, \tilde{U}$ and $\tilde{V}$ are arbitrary functions of a single variable. The integration of the two above differential equations $(27)$ and $(28)$ then gives

$$
\begin{aligned}
B(u, v) & =\frac{1}{2}\left(1+\lambda^{2}\right) A(u, v)+\frac{1}{2} \ln \left|f^{\prime}(\tilde{U}(u)+\tilde{V}(v))\right| \\
& +\frac{1}{2} \ln \left|\tilde{U}^{\prime}(u)\right|+\frac{1}{2} \ln \left|\tilde{V}^{\prime}(v)\right|+\zeta
\end{aligned}
$$

where $\zeta$ is a constant of integration, which can be chosen arbitrarily by appropriate rescaling (we will take $e^{2 \zeta}=4$ for convenience). The function $f$ is determined by reinserting (30) into the $(u v)$ component of Einstein's equations, equation (5), and one gets

$$
\tilde{U}^{\prime}(u) \tilde{V}^{\prime}(v)\left(f^{\prime \prime}+3 f^{\prime 2}-4 \mathcal{V}_{0} f^{\prime} e^{\left(1-\lambda^{2}\right) f}\right)=0
$$

with

$$
\mathcal{V}_{0}=\frac{\kappa^{2}}{6} V_{0}
$$

Taking $\tilde{U}^{\prime}(u)$ and $\tilde{V}^{\prime}(v)$ non zero (this is necessary if $V_{0} \neq 0$ ), this gives a second-order differential equation for $f$, which after a first integration yields

$$
\frac{\mathcal{V}_{0}}{1-\left(\lambda^{2} / 4\right)} e^{\left(4-\lambda^{2}\right) f}+\nu=f^{\prime} e^{3 f}
$$

where $\nu$ is an integration constant.

Once we know $f$, we can derive the metric everywhere

$$
d s^{2}=-\left|\tilde{U}^{\prime} \tilde{V}^{\prime} f^{\prime}\right| e^{f\left(1+\lambda^{2}\right)} d u d v+e^{2 f} \delta_{i j} d x^{i} d x^{j}
$$

This spacetime metric can in fact be written in a much simpler form, which turns out to be explicitly static. This is similar to the derivation, in [18], of the cosmological solutions with only a (negative) cosmological constant in the bulk, which are in fact Schwarzschild-AdS metrics. In order to see that, let us introduce new coordinates defined by

$$
\begin{aligned}
& R=e^{f} \\
& T=\tilde{U}-\tilde{V} .
\end{aligned}
$$


A straightforward substitution in the metric (34) yields the manifestly static metric

$$
d s^{2}=-h(R) d T^{2}+\frac{d R^{2}}{g(R)}+R^{2} \delta_{i j} d x^{i} d x^{j},
$$

with

$$
h(R)=-\frac{\mathcal{V}_{0}}{1-\left(\lambda^{2} / 4\right)} R^{2}-\nu R^{\lambda^{2}-2}
$$

and

$$
g(R)=-\frac{\mathcal{V}_{0}}{1-\left(\lambda^{2} / 4\right)} R^{2-2 \lambda^{2}}-\nu R^{-2-\lambda^{2}} .
$$

These solutions correspond to the type II solutions of [13], which were obtained by looking for moving branes in static background metrics. Here we did not assume beforehand staticity

of the background like in [13], but we have shown that the ansatz (25) necessarily leads to static solutions (by static here, we mean a Killing symmetry in the non-trival two dimensions, including the case of a spacelike Killing vector if $T$ is a space coordinate). This result is not so surprising since, with our ansatz (25), we have somehow 'frozen' the configuration of the scalar field with respect to the (bulk) scalar field. Note that, for $\lambda=0$, one recovers the standard result of brane cosmology without scalar field, $h(R)=g(R)=-\mathcal{V}_{0} R^{2}-\nu R^{-2}$, corresponding to a AdS-Schwarzschild metric if $\mathcal{V}_{0}$ is negative. The reason for this is that the scalar field is set to zero and its potential then acts as a cosmological constant.

\section{B. Brane motion and junction conditions}

The ansatz (25) confronted to the junction conditions (12) and (21) implies the following constraint on the matter coupling:

$$
\gamma=-\lambda
$$

It is now instructive to consider the junction conditions in the static coordinate system (37). The inconvenience of this coordinate system is that the brane cannot be considered at a fixed position. One must therefore study the motion of the brane, in order to express 
properly the junction conditions, which in turn will give us the cosmological evolution inside the brane. We just give the results here since this calculation can be found in the literature (see e.g. [13). The motion of the brane can be effectively described in the two-dimensional spacetime spanned by the coordinates $(T, R)$, where the brane behaves like a point with a trajectory given by $(T(\tau), R(\tau))$, where $\tau$ is the proper time of an observer comoving with the brane, i.e,

$$
d \tau^{2}=h(R) d T^{2}-\frac{d R^{2}}{g(R)}
$$

The components of the outward normal vector read

$$
n^{a}=\left(\frac{\dot{R}}{\sqrt{h g}}, \sqrt{g+\dot{R}^{2}}, 0,0,0\right)
$$

where, in this subsection (and only here), a dot stands for a derivative with respect to the proper time $\tau$. Applying the junction conditions, one ends up with

$$
\frac{\dot{R}^{2}}{R^{2}}=\frac{\kappa^{4}}{36} \rho^{2}-\frac{g(R)}{R^{2}}=\frac{\kappa^{4}}{36} \rho^{2}+\frac{\mathcal{V}_{0}}{1-\left(\lambda^{2} / 4\right)} R^{-2 \lambda^{2}}+\nu R^{-4-\lambda^{2}} .
$$

Note that here, although the background metric turns out to be the same as that found in [13], we consider more general cosmological situations because we do not restrict the equation of state of the matter on the brane to be that of a cosmological constant. In this picture, a different equation of state corresponds to a different trajectory in the static background spacetime.

\section{SEPARABLE SOLUTIONS}

In this section we look for exact solutions of Einstein's equations, which are separable when expressed in the light coordinate system, i.e. such that

$$
\begin{aligned}
& A(u, v)=A_{1}(u)+A_{2}(v), \\
& B(u, v)=B_{1}(u)+B_{2}(v), \\
& \phi(u, v)=\phi_{1}(u)+\phi_{2}(v) .
\end{aligned}
$$


To take advantage of this ansatz, one must consider an exponential potential

$$
V(\phi)=V_{0} e^{\alpha \phi}=V_{0} e^{\tilde{\alpha} \tilde{\phi}}, \quad \tilde{\alpha} \equiv \frac{\sqrt{3}}{\kappa} \alpha,
$$

so that the potential is the product of a function of $\phi_{1}$ with a function of $\phi_{2}$.

\section{A. Bulk equations}

With the above ansatz (44,47), all the second crossed derivatives in the bulk equations of motion disappear, and the system of differential equations (559) reduces to

$$
\begin{aligned}
A_{1}{ }^{\prime} A_{2}{ }^{\prime} & =\frac{\kappa^{2} V_{0}}{18} e^{2 B_{1}+2 B_{2}+\alpha \phi_{1}+\alpha \phi_{2}}, \\
2 A_{1}{ }^{\prime} B_{1}{ }^{\prime}-{A_{1}}^{\prime 2}-A_{1}{ }^{\prime \prime}-\frac{\kappa^{2}}{3}{\phi_{1}^{\prime}}^{2} & =0 \\
2 A_{2}{ }^{\prime} B_{2}{ }^{\prime}-{A_{2}}^{\prime 2}-A_{2}{ }^{\prime \prime}-\frac{\kappa^{2}}{3}{\phi_{2}}^{2} & =0 \\
6 A_{1}{ }^{\prime} A_{2}{ }^{\prime}+\kappa^{2} \phi_{1}^{\prime} \phi_{2}^{\prime} & =\frac{\kappa^{2} V_{0}}{2} e^{2 B_{1}+2 B_{2}+\alpha \phi_{1}+\alpha \phi_{2}}, \\
A_{2}{ }^{\prime} \phi_{1}^{\prime}+A_{1}{ }^{\prime} \phi_{2}^{\prime} & =-\frac{\alpha V_{0}}{6} e^{2 B_{1}+2 B_{2}+\alpha \phi_{1}+\alpha \phi_{2}},
\end{aligned}
$$

where the primes stand for ordinary derivatives, with respect to $u$ for quantities labelled by 1 and with respect to $v$ for quantities labelled with 2. It will be assumed from now on that $V_{0}$ is non zero. Comparison of (48) with (51) implies

$$
A_{1}^{\prime} A_{2}^{\prime}=\frac{\kappa^{2}}{3} \phi_{1}^{\prime} \phi_{2}^{\prime}
$$

hence the decomposition

$$
\begin{aligned}
& \tilde{\phi}_{1}^{\prime}(u)=C^{-1} A_{1}{ }^{\prime}(u), \\
& \tilde{\phi}_{2}^{\prime}(v)=C A_{2}{ }^{\prime}(v),
\end{aligned}
$$

where $C$ is a (non zero) separation constant (note that the cases where $\tilde{\phi}_{1}^{\prime}=0$ or $\tilde{\phi}_{2}^{\prime}=0$ are possible only if $V_{0}=0$ ). It turns out that this constant cannot be chosen arbitrarily. Indeed, substitution of (5455) into (52) and comparison with (48) imposes the following relation (if $V_{0}$ is non zero): 


$$
\tilde{\alpha}=-\frac{1+C^{2}}{C}
$$

which implies that $|\tilde{\alpha}| \geq 2$. The first equation of the system, (48), can also be separated, giving the two following relations,

$$
\begin{aligned}
e^{2 B_{1}} & =D e^{-\tilde{\alpha} \tilde{\phi}_{1}} \tilde{\phi}_{1}^{\prime}, \\
e^{2 B_{2}} & =\frac{18}{\kappa^{2} V_{0} D} e^{-\tilde{\alpha} \tilde{\phi}_{2}} \tilde{\phi}_{2}^{\prime},
\end{aligned}
$$

where $D$ is a (non zero) separation constant, and where the $A_{i}$ have been replaced by the

$\tilde{\phi}_{i}$ using (54-55). It is then easy to check that the remaining equations (49) and (50) are automatically satisfied, since after use of (54) and (55), they are simply the derivatives of the expressions (57) and (58).

We have thus solved completely the Einstein equations in the bulk. Our solutions are parametrized by two arbitrary functions $A_{1}$ and $A_{2}$, from which all the components of the metric are obtained. Let us now turn to the boundary conditions at the brane location.

\section{B. Junction conditions}

We must now make the link between the metric in the bulk and the matter in the brane. This comes from the junction conditions (12-13) and (21). Comparison between the junction conditions (12) and (21) leads to the following relation

$$
A_{2}^{\prime}(t)=\beta A_{1}^{\prime}(t)
$$

with

$$
\beta \equiv \frac{C \gamma+1}{C(C+\gamma)}
$$

which can be integrated immediately if one assumes that $\gamma$ is constant, i.e. that $w$ is constant (note that $C+\gamma \neq 0$ otherwise $A_{1}^{\prime}=0$ which is forbidden for $V_{0} \neq 0$ ).

Comparison of the junction condition (13) for $B$ with the junction condition (12) for $A$, in which one replaces $B_{1}$ and $B_{2}$ with their respective expression in terms of $A_{1}$ and 
$A_{2}$ according to (57-58) and (54-55), leads to a more complicated expression involving the second derivatives of $A_{1}$ and $A_{2}$, which reads

$$
\left[-\frac{\tilde{\alpha}}{C}+2(3 w+2)\right] A_{1}^{\prime}-[-\tilde{\alpha} C+2(3 w+2)] A_{2}^{\prime}=-\frac{A_{1}^{\prime \prime}}{A_{1}^{\prime}}+\frac{A_{2}^{\prime \prime}}{A_{2}^{\prime}},
$$

where the $A_{i}$ are here only functions of time $t$ because we are on the brane, i.e. at $y=0$. This expression can be rewritten in the form

$$
\left[\left(-\frac{\tilde{\alpha}}{C}+2(3 w+2)\right)-(-\tilde{\alpha} C+2(3 w+2)) \beta\right] A_{1}^{\prime}=\frac{\beta^{\prime}}{\beta} .
$$

For simplicity, we will restrict ourselves to the cases where the equation of state is such that $w$ is constant, which implies in turn that $\gamma$ is constant. In this case, the right hand side of (62) must vanish because of (60), and one finds the following relation between the constants $C, \gamma$ and $\alpha$ :

$$
\left(C^{2}-1\right)(\tilde{\alpha} \gamma+2(2+3 w))=0
$$

where one must recall that $\tilde{\alpha}$ is a function of $C$, given in (56). This condition can be satisfied if $C= \pm 1$ or if

$$
\gamma=-\frac{2(2+3 w)}{\tilde{\alpha}}
$$

As it will be clear below (see equation( $[69)$ ), the cases $C= \pm 1$ (corresponding to $\tilde{\alpha}=\mp 2$ by (56)) are not very interesting since they lead to a zero energy density in the brane, which means that the brane is only virtual and does not really exist physically. For the, more interesting, other cases, the condition we obtain tells us that for a given potential, i.e. for a given choice of the coefficient $\tilde{\alpha}$ (with $|\tilde{\alpha}| \geq 2$ ), the coupling between the bulk scalar field and the brane matter is enterely determined by the equation of state if one wishes to find separable solutions.

After having established all conditions required by the compatibility between the three junction conditions, one can now compute the various quantities on the brane in terms of $A(t)$ only. Indeed, starting from (see equation (59)) 


$$
A(t)=(1+\beta) A_{1}(t)
$$

where the integration constant can be ignored, up to a coordinate rescaling, one finds for

the scalar field, using (5455) (and ignoring once more the integration constants which can be reabsorbed in a rescaling of $V_{0}$ ),

$$
\tilde{\phi}(t)=C^{-1} A_{1}(t)+C A_{2}(t)=\frac{2-\gamma \tilde{\alpha}}{2 \gamma-\tilde{\alpha}} A(t) .
$$

The other metric component follows from (5758) is given by

$$
e^{B}(t)=\sqrt{\frac{18 \beta}{\kappa^{2} V_{0}}} \exp \left[-\frac{\tilde{\alpha}}{2}\left(C^{-1}+\beta C\right) A_{1}\right]\left|A_{1}^{\prime}\right| .
$$

Finally, the energy density can be evaluated from (12). Inserting the product of (57) and (58), one finds

$$
\frac{\kappa^{2}}{6} \rho=e^{-B}(1-\beta) A_{1}^{\prime}= \pm \sqrt{\frac{\kappa^{2} V_{0}}{18 \beta}}(1-\beta) e^{\tilde{\alpha} \tilde{\phi} / 2},
$$

which eventually gives

$$
\rho= \pm \sqrt{\frac{2 V_{0}\left(\tilde{\alpha}^{2}-4\right)}{\kappa^{2}\left(1-\tilde{\alpha} \gamma+\gamma^{2}\right)}} e^{\tilde{\alpha} \tilde{\phi} / 2} .
$$

We will explore in more details the corresponding brane cosmology in the next section.

\section{COSMOLOGICAL EVOLUTION IN THE BRANE}

The purpose of this section is to analyse the cosmological behaviour inside the brane, given the global solutions obtained in the previous section. The brane being assumed to stay at $y=0$, the induced metric in the brane is simply

$$
d s^{2}=-e^{2 B(t, 0)} d t^{2}+e^{2 A(t, 0)} \delta_{i j} d x^{i} d x^{j}
$$

The cosmological scale factor thus corresponds to

$$
a(t)=e^{A(t, 0)}
$$

and the cosmic time $\tau$ can be derived from the time $t$ by

$$
\tau=\int e^{B(t, 0)} d t
$$




\section{A. Generalized Friedmann equations}

As a first step, we will combine some of the Einstein equations evaluated on the brane with the junction conditions, and thus establish a generalized Friedmann's equation as well as a generalized conservation equation. In order to do so, we will closely follow the derivation of [4] (and use the notation $a(t, y) \equiv e^{A(t, y)}$ ) with the additional ingredient that we allow here for an energy flux from the fifth dimension, i.e. the component $(0,5)$ of the bulk energymomentum tensor is non zero because of the presence of the scalar field. We do not rewrite here the components of the Einstein tensor in the $(t, y)$ coordinate system but we refer the reader to [4] where they are explicitly written.

Substitution of the junction conditions in the $(0,5)$ component of the Einstein's equations evaluated at the brane, immediately yields the generalized conservation equation

$$
\dot{\rho}+3 \frac{\dot{a}}{a}(\rho+p)=\mathcal{F}
$$

with

$$
\mathcal{F}=\left.2 e^{-B} T_{05}\right|_{y=0}
$$

Here $T_{05}=\dot{\phi} \phi^{\prime}$, and using the junction condition for the scalar field, one ends up with the equation

$$
\dot{\rho}+3 \frac{\dot{a}}{a}(\rho+P)=\gamma \dot{\tilde{\phi}} \rho
$$

This generalizes the usual conservation law for cosmological matter, for wich the right hand side is zero. The integration of the equation yields the following evolution for the energy density for an equation of state $P=w \rho$ with $w$ constant:

$$
\rho \propto a^{-3(1+w)} e^{\gamma \tilde{\phi}}
$$

and one recovers the familiar evolution of standard cosmology only if the scalar field is constant in time. 
Let us now consider the $(5,5)$ component of the Einstein equations. Using the $(0,5)$ component, it can be rewritten in the form

$$
\dot{F}=\frac{2}{3} \dot{a} a^{3} \kappa^{2} T_{5}^{5}-\frac{2}{3} a^{\prime} a^{3} \kappa^{2} T_{0}^{5}
$$

with

$$
F \equiv e^{-2 B}\left[\left(a a^{\prime}\right)^{2}-(a \dot{a})^{2}\right]
$$

This corresponds to a slight generalization of the expression given in 4, allowing here for a non vanishing flux from the extra-dimension. Taking the value of (77) at the brane, and using the junction condition, one finds after integration in time, the following generalized Friedmann's equation

$$
H^{2}=\frac{\kappa^{4}}{36} \rho^{2}-\frac{2}{3 a^{4}} \int d \tau\left(\frac{d a}{d \tau}\right) a^{3} \kappa^{2} T_{5}^{5}-\frac{\kappa^{4} \gamma}{18 a^{4}} \int d \tau a^{4}\left(\frac{d \tilde{\phi}_{0}}{d \tau}\right) \rho^{2}
$$

where the Hubble parameter is defined by

$$
H \equiv \frac{d a / d \tau}{a}=e^{-B} \frac{\dot{a}}{a}
$$

This equation can be found, with a slightly different presentation, in [16]. It is characterized by the quadratic appearance of the energy density of the brane, which is a generic feature of brane cosmology [1]. We also have an integral term related to the pressure along the fifth dimension and an integral term related to the energy flux coming from the fifth dimension, which here is essentially related to the time variation on the brane of the bulk scalar field.

Finally, the expression for the energy-momentum tensor of the scalar field gives us

$$
T_{5}^{5}=\frac{1}{2} e^{-2 B}\left(\phi^{\prime 2}+\dot{\phi}^{2}\right)-V(\phi)
$$

which yields

$$
\left.\frac{2}{3} \kappa^{2} T_{5}^{5}\right|_{y=0}=\frac{\kappa^{4}}{36} \gamma^{2} \rho^{2}+\left(\frac{d \tilde{\phi}_{0}}{d \tau^{2}}\right)^{2}-\frac{2}{3} \kappa^{2} V_{0} e^{\alpha \tilde{\phi}_{0}}
$$

where we have used the junction condition (21) to replace the gradient contribution by the term quadratic in the energy density. 


\section{B. Static bulk solutions}

The generalized Friedmann equation has the form

$$
H^{2}=\frac{\kappa^{2}}{36} \rho^{2}+\frac{\mathcal{V}_{0}}{1-\left(\lambda^{2} / 4\right)} a^{-2 \lambda^{2}}+\nu a^{-4-\lambda^{2}} .
$$

Combining this Friedmann equation with the conservation equation, one can obtain explicitly

the evolution of the scale factor as a function of the cosmic time for any given equation of state.

It is easy to check that the general form (79) for the Friedmann equation is compatible with the above Friedmann equation. The five-dimensional pressure can be expressed in the form

$$
\left.\frac{2}{3} \kappa^{2} T_{5}^{5}\right|_{y=0}=\frac{\kappa^{4}}{18} \lambda^{2} \rho^{2}-\lambda^{2} \frac{g(a)}{a^{2}}-\frac{2}{3} \kappa^{2} V_{0} a^{-2 \lambda^{2}}
$$

where we have used the Friedmann's equation in the form (83) to replace the kinetic term. Substituting in the integral terms, one finds that the two $\rho^{2}$ terms just cancel, and after integration of the remaining terms, one obtains directly what one expects, i.e.

$$
\frac{2}{3 a^{4}} \int d \tau \dot{a} a^{3} \kappa^{2} T_{5}^{5}+\frac{\kappa^{4}}{18 a^{4}} \int d \tau a^{4} \gamma \dot{\tilde{\phi}} \rho^{2}=\frac{g(a)}{a^{2}} .
$$

\section{Separable solutions}

According to (72) and (67), the cosmic time is given by

$$
\tau=\sqrt{\frac{18 \beta}{\kappa^{2} V_{0}}} \mu^{-1} e^{\mu A_{1}} \equiv \tau_{0} e^{\mu A_{1}}, \quad \mu=\frac{\tilde{\alpha}(\tilde{\alpha} \gamma-2)}{2(\gamma+C)}
$$

where we have assumed $A_{1}^{\prime}>0$ for definiteness. The scale factor is given by

$$
a(\tau)=\left(\frac{\tau}{\tau_{0}}\right)^{p}, \quad p \equiv \frac{1+\beta}{\mu}=\frac{2(\tilde{\alpha}-2 \gamma)}{\tilde{\alpha}(2-\gamma \tilde{\alpha})} .
$$

Substituting the condition (64) for the coupling $\gamma$, in the interesting case where the brane is not empty, the power can be written 


$$
p=\frac{1}{3(1+w)}\left[1+\frac{4(2+3 w)}{\tilde{\alpha}^{2}}\right] .
$$

The minimum value of $\tilde{\alpha}^{2}$ being 4 , the range of possible $p$ is between $p=1$ and $p=$ $1 /(3(1+w))$, the latter being the unconventional evolution in the case of an empty bulk [1]. Note that this range includes the conventional value $p=2 /(3(1+w))$.

The Hubble parameter is therefore

$$
H=\frac{p}{\tau}
$$

The energy density in the brane can be related to this Hubble paramter, the easiest way being to use (68) and one finds that the two are proportional,

$$
\frac{\kappa^{2}}{6} \rho=\frac{1-\beta}{1+\beta} H
$$

The unconventional cosmological evolution that was obtained in the case of an empty bulk [1] is thus generalized here for a bulk with a scalar field for our specific separable solutions. There is a difference however is the proportionality coefficient. Finally, the evolution of the scalar field is given by

$$
\tilde{\phi}_{0}=\frac{2-\gamma \tilde{\alpha}}{2 \gamma-\tilde{\alpha}} \ln a \text {. }
$$

It is now instructive to check the generalized Friedmann equation (79), by evaluating the contribution of each term for our explicit solutions. One finds

$$
-\frac{2}{3 a^{4}} \int d \tau\left(\frac{d a}{d \tau}\right) a^{3} \kappa^{2} T_{5}^{5}=-2 \frac{\tilde{\alpha}^{2} \gamma^{2}+4 \tilde{\alpha} \gamma-8 \gamma^{2}-4}{(\tilde{\alpha}-2 \gamma)\left(\tilde{\alpha}^{2} \gamma+2 \tilde{\alpha}-8 \gamma\right)} \frac{p^{2}}{\tau^{2}}
$$

and

$$
-\frac{\kappa^{4} \gamma}{18 a^{4}} \int d \tau a^{4}\left(\frac{d \tilde{\phi}_{0}}{d \tau}\right) \rho^{2}=-2 \gamma \frac{\left(\tilde{\alpha}^{2}-4\right)(\tilde{\alpha} \gamma-2)}{(\tilde{\alpha}-2 \gamma)^{2}\left(\tilde{\alpha}^{2} \gamma+2 \tilde{\alpha}-8 \gamma\right)} \frac{p^{2}}{\tau^{2}}
$$

and the sum of these two terms indeed coincides with

$$
H^{2}-\frac{\kappa^{4}}{36} \rho^{2}=4 \frac{1-\tilde{\alpha} \gamma+\gamma^{2}}{(\tilde{\alpha}-2 \gamma)^{2}} H^{2}
$$


These expressions can be rewritten, using (64), in terms of the parameter $w$ of the equation of state. One finds

$$
\begin{gathered}
-\frac{2}{3 a^{4}} \int d \tau\left(\frac{d a}{d \tau}\right) a^{3} \kappa^{2} T_{5}^{5}=4 \frac{\left(9 w^{2}+6 w-1\right) \tilde{\alpha}^{2}-8\left(9 w^{2}+12 w+4\right)}{\left(8+12 w+\tilde{\alpha}^{2}\right)\left((3 w+1) \tilde{\alpha}^{2}-8(3 w+2)\right)} \frac{p^{2}}{\tau^{2}} \\
-\frac{\kappa^{4} \gamma}{18 a^{4}} \int d \tau a^{4}\left(\frac{d \tilde{\phi}_{0}}{d \tau}\right) \rho^{2}=12 \tilde{\alpha}^{2} \frac{(1+w)(2+3 w)\left(\tilde{\alpha}^{2}-4\right)}{\left(8+12 w+\tilde{\alpha}^{2}\right)^{2}\left((3 w+1) \tilde{\alpha}^{2}-8(3 w+2)\right)} \frac{p^{2}}{\tau^{2}},
\end{gathered}
$$

and

$$
H^{2}-\frac{\kappa^{4}}{36} \rho^{2}=4 \frac{(5+6 w) \tilde{\alpha}^{2}+4(2+3 w)^{2}}{\left(8+12 w+\tilde{\alpha}^{2}\right)^{2}} H^{2} .
$$

Let us also mention the particular case $\gamma \tilde{\alpha}=2$, corresponding to the equation of state $w=-1$. One can easily see that the energy density is constant, given by

$$
\rho=\sqrt{-\frac{4 \tilde{\alpha}^{2} V_{0}}{\kappa^{2}}}
$$

which implies in particular that the scalar field potential must be negative for consistency. The scale factor, is given, instead of a power-law, by an exponential, namely

$$
a(\tau)=\exp \left[\sqrt{-\frac{\kappa^{2} V_{0}}{18}\left(\tilde{\alpha}^{2}-4\right)} \tau\right]
$$

\section{Cosmology with conformal coupling}

In the case of conformal coupling, one must be aware that the cosmological evolution given above corresponds to the so-called Einstein frame, i.e. the frame associated with the metric for which the action is the usual Einstein-Hilbert action (here we extend this definition to the five -dimensional gravitational action). However, the physical metric corresponds to the metric which is minimally coupled to the ordinary matter and in our case, this means that the physical frame, usually called the Jordan frame in the context of scalar-tensor theories of gravitation, is the frame associated with the metric $\tilde{g}_{\mu \nu}$.

It is therefore useful to rewrite the previous equations in the physical frame. The correspondance between the two scale factors is 


$$
\tilde{a}=e^{\chi \tilde{\phi}} a
$$

The proper times are also different and related by

$$
d \tilde{\tau}=e^{\chi \tilde{\phi}} d t
$$

Using the relation (91), one finds that

$$
e^{\tilde{\phi}}=\left(\frac{\tau}{\tau_{0}}\right)^{-2 / \tilde{\alpha}}
$$

and therefore, the expression of the new proper time $\tilde{\tau}$ in terms of the "Einstein frame" proper time is

$$
\tilde{\tau}=\tilde{\tau}_{0}\left(\frac{\tau}{\tau_{0}}\right)^{1-\frac{2 \chi}{\tilde{\alpha}}}, \quad \tilde{\tau}_{0} \equiv \frac{\tau_{0}}{1-\frac{2 \chi}{\tilde{\alpha}}} .
$$

The cosmological evolution of the scale factor is thus given by

$$
\tilde{a}=\left(\frac{\tilde{\tau}}{\tilde{\tau}_{0}}\right)^{\left(p-\frac{2 \chi}{\tilde{\alpha}}\right) /\left(1-\frac{2 \chi}{\tilde{\alpha}}\right)} .
$$

Note that one cannot find a separable solution with conformal coupling in the case of radiation. Conformal coupling, according to (22), implies $\gamma=0$ for radiation, which is clearly incompatible with the condition (64) for separable solutions.

\section{CONCLUSIONS}

In the present work, we have obtained explicit exact solutions for a five-dimensional spacetime with a scalar field and a 3-brane. Although several explicit solutions exist already in the literature, most of them correspond to static solutions for which the bulk geometry and scalar field are frozen and the cosmological evolution is only due to the motion of our brane-universe in this bulk. As we have shown here, this is the case when one assumes some proportionality relation between the scalar field and the logarithm of the bulk scale factor. More interestingly, we have also found solutions where the bulk itself has some dynamics. Even if our solutions are rather artificial, and cannot be considered as realistic models for 
the recent cosmology of our universe, they might be useful to give some insights in the future investigations of bulk-brane dynamics.

Although these solutions might represent acceptable models for the very early universe, one of the main open problems is to obtain generic solutions of the bulk-brane system. One could then investigate whether there exist, or not, some attractor solutions so that the primordial brane cosmology would be somewhat insensitive to the initial conditions.

Another open question is how, in a cosmological context, the presence of the scalar field and of its perturbations might affect the results obtained so far for cosmological perturbations in a brane-universe, where the bulk was assumed to be empty apart the presence of a negative cosmological constant (see [19] and references therein).

Acknowledgments: we would like to thank S. Carroll for interesting discussions, as well as C. Charmousis for his useful comments on the present work and for informing us about his work in progress, where he has obtained, independently, and using a similar technique, some of the results presented here. 


\section{REFERENCES}

[1] P. Binétruy, C. Deffayet, D. Langlois, Nucl. Phys. B 565, 269 (2000) hep-th/9905012.

[2] C. Csáki, M. Graesser, C. Kolda, J. Terning, Phys. Lett. B 462, 34 (1999) hepph/9906513]; J.M. Cline, C. Grojean, G. Servant, Phys. Rev. Lett. 83, 4245 (1999) hep-ph/9906523.

[3] L. Randall, R. Sundrum, Phys. Rev. Lett. 83, 4690 (1999) hep-th/9906064.

[4] P. Binétruy, C. Deffayet, U. Ellwanger, D. Langlois, Phys.Lett. B 477, 285 (2000) hep-th/9910219.

[5] T. Shiromizu, K. Maeda, M. Sasaki, Phys. Rev. D 62, 024012 (2000) gr-qc/9910076; E. Flanagan, S. Tye, I. Wasserman, Phys. Rev. D 62, 044039 (2000) hep-th/9910498

[6] W.D. Goldberger, M.B. Wise, Phys. Rev. Lett 83, 4922 (1999) hep-ph/9907447; O. DeWolfe, D.Z. Freedman, S.S. Gubser, A. Karch, Phys. Rev. D62, 046008 (2000) thepth/9909134; T. Tanaka, X. Montes, Nucl. Phys. B 582, 259 (2000) hep-th/0001092

[7] L. Randall, R. Sundrum, Phys. Rev. Lett. 83, 3370 (1999) hep-ph/9905221.

[8] N. Arkani-Hamed, S. Dimopoulos, N. Kaloper, R. Sundrum, Phys. Lett. B 480, 193 (2000) hep-th/0001197]; S. Kachru, M. Schulz and E. Silverstein, Phys. Rev. D 62, 045021 (2000) hep-th/0001206

[9] G.T. Horowitz, I. Low, A. Zee, Phys. Rev. D 62086005 (2000) hep-th/0004206

[10] P. Binétruy, J.M. Cline, C. Grojean, Phys. Lett. B 489403 (2000) hep-th/0007029

[11] P. Brax, A. Davis JHEP 0105007 (2001) hep-th/0104023

[12] A. Feinstein, K.E. Kunze, M.A. Vazquez-Mozo, Curved dilatonic brane worlds, hepth/0105182

[13] H.A. Chamblin, H.S. Reall, Nucl. Phys. B 562133 (1999) hhep-th/9903225] 
[14] K. Maeda, D. Wands, Phys. Rev. D 62124009 (2000) hep-th/0008188

[15] A. Mennim, R.A. Battye, Class. Quant. Grav. 182171 (2001) hep-th/0008192

[16] C. van de Bruck, M. Dorca, C.J.A.P. Martins, M. Parry, Phys. Lett. B 495183 (2000) hep-th/0009056

[17] S. Carroll, L. Mersini, Can we live in a self-tuning universe ?, hep-th/0105007

[18] P. Bowcock, C. Charmousis, R. Gregory, Class. Quant. Grav. 17, 4745 (2000) hepth/0007177

[19] D. Langlois, Phys. Rev. Lett. 86, 2212 (2001) 